\title{
Molten globule-triggered inactivation of a thermostable and solvent stable lipase in hydrophilic solvents.
}

\begin{abstract}
The use of lipase in hydrophilic solvent is usually hampered by inactivation. The solvent stability of a recombinant solvent stable lipase isolated from thermostable Bacillus sp. strain 42 (Lip 42), in DMSO and methanol were studied at different solvent-water compositions. The enzymatic activities were retained in up to $45 \% \mathrm{v} / \mathrm{v}$ solvent compositions. The near-UV CD spectra indicated that tertiary structures were perturbed at $60 \%$ v/v and above. Far-UV $\mathrm{CD}$ in methanol indicated the secondary structure in Lip 42 was retained throughout all solvent compositions. Fluorescence studies indicated formations of molten globules in solvent compositions of $60 \% \mathrm{v} / \mathrm{v}$ and above. The enzyme was able to retain its secondary structures in the presence of methanol; however, there was a general reduction in $\beta$-sheet and an increase in $\alpha$-helix contents. The H-bonding arrangements triggered in methanol and DMSO, respectively, caused different forms of tertiary structure perturbations on Lip 42, despite both showing partial denaturation with molten globule formations.
\end{abstract}

Keyword: Molten globule; Solvent stable lipase; Hydrophilic solvent; Overexpression; Thermostable lipase. 